\title{
Marginal zone B cells emerge as a critical component of pregnancy well-being
}

\author{
Damián O Muzzio ${ }^{1}$, Katharina B Ziegler ${ }^{1}$, Jens Ehrhardt ${ }^{1}$, Marek Zygmunt ${ }^{1}$ \\ and Federico Jensen ${ }^{1,2,3}$ \\ ${ }^{1}$ Research Laboratory, Department of Obstetrics and Gynecology, University of Greifswald, Greifswald, Germany, \\ ${ }^{2}$ Laboratory for Immunology of Pregnancy, Center for Pharmacological and Botanical Studies (CEFYBO-CONICET- \\ UBA), Paraguay 2155 Floor 17th, Buenos Aires C1121ABG, Argentina and ${ }^{3}$ Institute of Health Sciences, National \\ University Arturo Jauretche, Buenos Aires, Argentina
}

Correspondence should be addressed to Federico Jensen; Email: fjensen@unaj.edu.ar or federico.jensen@outlook.com

\begin{abstract}
The success of eutherian mammal evolution was certainly supported by the ability of the already existing immune system to adapt to the presence of the semi-allogeneic fetus without losing the capability to defend the mother against infections. This required the acquisition of highly regulated and coordinated immunological mechanisms. Failures in the development of these strategies not only lead to the interruption of pregnancy but also compromise maternal health. Alongside changes on the cytokine profile - expansion of tolerogenic dendritic and regulatory $T$ cells - a profound adaptation of the B cell compartment during pregnancy was recently described.

Among others, the suppression of B cell lymphopoiesis and B cell lymphopenia were proposed to be protective mechanisms tending to reduce the occurrence of autoreactive $B$ cells that might recognize fetal structures and put pregnancy on risk. On the other hand, expansion of the pre-activated marginal zone (MZ) B cell phenotype was described as a compensatory strategy launched to overcome B cell lymphopenia thus ensuring a proper defense. In this work, using an animal model of pregnancy disturbances, we demonstrated that the suppression of B cell lymphopoiesis as well as splenic B cell lymphopenia occur independently of pregnancy outcome. However, only animals undergoing normal pregnancies, but not those suffering from pregnancy disturbances, could induce an expansion and activation of the MZ B cells. Hence, our results clearly show that MZ B cells, probably due to the production of natural protective antibodies, participate in the fine balance of immune activation required for pregnancy well-being.

Reproduction (2016) 151 29-37
\end{abstract}

\section{Introduction}

The evolution of the eutherian mammal has surely represented a challenge to the rules of the already existing immune system, as it faced the dilemma to simultaneously tolerate the presence of a semiallogeneic fetus without losing the capacity to defend the mother against pathogens. This dichotomy seems to have been resolved by the acquisition of highly regulated immune strategies ensuring a fine balance between immune suppression, allowing the presence of the fetus, and immune activation, guaranteeing a proper defense.

Alongside well-known and deeply investigated immune adaptations involving dendritic cells (Segerer et al. 2012), T cells (Ruocco et al. 2014), NK cells (Parham 2004) and macrophages (Faas et al. 2014), the B cell compartment was recently demonstrated to undergo profound modifications and adaptations during pregnancy.
B lymphocytes are fundamental components of the adaptive arm of the immune system. B cells are continuously originated from pluripotent hematopoietic stem cells in the bone marrow (BM). The pro- and pre-B cells represent the earliest $B$ cell stages and are characterized by low expression levels of B220 and the absence of IgM expression on their cellular membrane (Hardy \& Hayakawa 1991, Hardy 2003). The transition from pro- to pre-B cell stage requires the loss of expression of the sialoglycoprotein CD43. Once pre-B cells start expressing IgM on their cellular membrane, they become immature B cells, leave the BM and migrate to the spleen where they continue their development (Hardy 2003). In this tissue, immature B cells give rise to either follicular (FO) or marginal zone (MZ) B cells, the most prominent mature $B$ cell subsets in the body (Allman \& Pillai 2008). These two B cell populations have major differences in terms of phenotype, localization and functionality. FO B cells, localized in the follicular niche of the spleen and lymph nodes, are 
defined as B220 ${ }^{+} \mathrm{CD} 23^{\text {hi }} \mathrm{CD} 21^{\text {int }}$ (Allman \& Pillai 2008, Chu et al. 2008). Once encountering an antigen, FO B cells move into the germinal center and, upon additional signals given by $\mathrm{T}$ cells, differentiate into high affinity IgG-producing plasma cells and memory B cells (Allman \& Pillai 2008, Chu et al. 2008). This process lasts $\sim 5$ days, a time that can be crucial especially for fast replicating pathogens. However, this gap is covered by the $\mathrm{B} 220^{+} \mathrm{CD} 23^{\mathrm{lo}} \mathrm{CD} 21^{\mathrm{hi}} \mathrm{MZ}$ B cells that, due to their pre-activated phenotype and strategic localization in the marginal sinus area of the spleen, rapidly respond against pathogens, giving rise to short-life plasma cells producing low affinity antibodies (Cerutti et al. 2013). MZ B cells are also involved in the production of IgM and $\operatorname{IgA}$ natural antibodies that control the first wave of an infection.

In previous studies, we and others have demonstrated that pregnancy induces a strong suppression of B cell lymphopoiesis and proposed that this might represent an evolutionarily acquired mechanism tending to reduce the occurrence of autoreactive $B$ cells that may recognize fetal structures causing pregnancy failures (Medina et al. 1993, Ait-Azzouzene et al. 1998, Muzzio et al. 2014). Despite B cell lymphopenia, we also showed that pregnant mice display an expansion of the pre-activated MZ B cells, suggesting an attempt to compensate for the reduced number of $\mathrm{B}$ cells during gravidity thus ensuring a proper defense (Muzzio et al. 2014).

In this study, using a well-established animal model of immune-mediated pregnancy failures, we aimed to deeply investigate the participation of $\mathrm{B}$ cells in pregnancy outcome.

\section{Materials and methods}

\section{Animals}

Eight-week-old female $\mathrm{CBA} / \mathrm{j}\left(\mathrm{H} 2^{\mathrm{k}}\right)$ as well as $\mathrm{DBA} / 2 \mathrm{~J}$ $\left(\mathrm{H} 2^{\mathrm{d}}\right)$ and $\mathrm{BALB} / \mathrm{C}\left(\mathrm{H} 2^{\mathrm{d}}\right)$ males were purchased from Charles River (Sulzfeld, Babavia, Germany). All mice were maintained in the facilities of the BioTechnikum Greifswald under a $12 \mathrm{~h}$ light: $12 \mathrm{~h}$ darkness cycle with free access to water and chow. Animal experiments were carried out according to institutional guidelines after ministerial approval (institutional review board: Landesverwaltungsamt Sachsen-Anhalt (ID: FJ2-1019 to F J) and Landesamt für Landwirtschaft, Lebensmittelsicherheit und Fischerei Mecklenburg-Vorpommern (7221.3$1-068 / 13$ to $F \mathrm{~J})$ ). The experiments were conducted in conformity with the European Communities Council Directive 86/609/EEC.

For our study, we used a well-established model of immune-mediated pregnancy disturbances (Clark et al. 1986). Shortly after CBA/J females were mated to DBA/2 J males, a median of $20-30 \%$ of the embryos resorbed (aborted). In addition, DBA/2J pregnant CBA females also displayed angiogenic deregulation, abnormal placental development and fetal growth restriction, all features of pre-eclampsia (Kanasaki \& Kalluri 2009, Ahmed et al. 2010). BALB/c-mated CBA/J females represented the normal pregnancy combination with a median of $0 \%$ abortions and none of the symptoms described above for the DBA/2J-mated $\mathrm{CBA} / \mathrm{J}$ females. Age-matched virgin $\mathrm{CBA} / \mathrm{J}$ females were mated with $\mathrm{BALB} / \mathrm{c}$ or DBA/2J males. Females were inspected daily for vaginal plugs, and the presence of a vaginal plug was designated as day 0 of pregnancy. Pregnant females were sacrificed at day 14 of pregnancy.

\section{Cell preparation and flow cytometry}

For flow cytometry analysis, single cell suspensions were obtained from the isolated samples following previously described procedures (Muzzio et al. 2014). Briefly, cells were isolated from BM of the femur and tibia, spleen, blood and para-aortic lymph nodes that drain the uterus. Plasma was separated from heparinized whole blood after $10 \mathrm{~min}$ centrifugation at $1300 \mathrm{~g}$ and stored at $-80^{\circ} \mathrm{C}$ for further analysis. Organs were crushed in a $100 \mu \mathrm{m}$ cell strainer to obtain a single cell suspension and red blood cells were lysed for $5 \mathrm{~min}$. After washing, cell suspensions were counted using a Neubauer chamber, and $1 \times 10^{6}$ cells were stained 30 min at $4{ }^{\circ} \mathrm{C}$ with specific labeled antibodies (Table 1) or isotype controls. It is important to note that, although CD45R (B220) is mainly expressed by the B cell lineage from early pro-B to mature $B$ cells and it has been classically used as a B cell marker, according to eBioscience, it might also be expressed in some activated $\mathrm{T}$ cells, lymphokine activated killer cells, NK cell progenitors in the BM and T cells of the lpr/lpr mutant mouse. Therefore, in this study, apart from the analysis of total numbers of B cells in the spleen, this antibody has always been used in combination with other B cells markers. Data were acquired on FACSCalibur or FACS Canto (BD Biosciences, Hidelberg, Baden-Württemberg, Germany) and analyzed by using Flowlo software (Tree Star, Inc., Ashland, OR, USA). B cell populations were defined using gating strategies and the percentage was later referred to the cell count obtained by the

Table 1 Antibodies used in FACS analysis of the B cell populations in $\mathrm{CBA} / \mathrm{J}$ mice.

\begin{tabular}{llll}
\hline Molecule & Antibody clone & Label & $\begin{array}{l}\text { Alternate } \\
\text { nomeclature }\end{array}$ \\
\hline CD23 & B3B4 & PE & FCERII \\
CD21 & $7 \mathrm{G} 6$ & FITC & \\
CD19 & $1 \mathrm{~d} 3$ & PE-Cy7 & \\
CD43 & R2/60 & FITC & \\
CD45R & RA3-6B2 & PE-Cy7 & B220 \\
IgM & II/41 & APC & \\
IgD & $11-26 c$ & PE & \\
CD93 & AA4.1 & FITC & C1qRp \\
\hline
\end{tabular}

FACS, fluorescence-activated cell sorting. 
Neubauer chamber to obtain the absolute numbers of cells (Table 2).

\section{Analysis of immunoglobulin in serum}

Concentrations of $\lg M, \lg G 1, \lg \mathrm{G} 2 \mathrm{a}, \lg \mathrm{G} 2 \mathrm{~b}, \lg \mathrm{G} 3, \lg \mathrm{E}$ and IgA in plasma were quantified using the ProcartaPlex Mouse Antibody Isotyping Panel (eBioscience/Affymetrix, Frankfurt am Main, Hessen, Germany) and subsequently analyzed on Luminex test equipment (Bio-Plex® 200 Systems (Bio-Rad, Munich, Bavaria, Germany)). Before starting the assay, frozen plasma samples were thawed and centrifuged at $4{ }^{\circ} \mathrm{C}$ for $10 \mathrm{~min}$ at $1000 \mathrm{~g}$. A 1:10 000 dilution of the samples was made and the multiplex assay was performed on a 96-well filter plate as described in the manufacture's instructions. The standard curve range was as follows: IgG1 $(0.69-500 \mathrm{ng} / \mathrm{ml})$, IgG2a $(5.49-4000 \mathrm{ng} / \mathrm{ml}), \quad \operatorname{lgG} 2 \mathrm{~b}(4.12-3000 \mathrm{ng} / \mathrm{ml})$, IgG3 (10.25-7500), IgA $(4.12-3000 \mathrm{ng} / \mathrm{ml})$, IgE $(0.69-500 \mathrm{ng} / \mathrm{ml})$ and $\operatorname{lgM}(4.12-3000 \mathrm{ng} / \mathrm{ml})$.

\section{BAFF ELISA}

Levels of the B-cell activating factor (BAFF) of the TNF family in serum were assayed using a commercially available ELISA kit from R\&D Systems (Minneapolis, $M N$, USA), following the supplier's recommendations.

\section{Statistical analysis}

Data were analyzed with PRISM software (v. 5.01; GraphPad, La Jolla, CA, USA). To evaluate the differences of means among the groups, ANOVA followed by Tukey's multiple comparison test or Kruskal-Wallis test were used. Significant differences are indicated with asterisks $\left({ }^{*} P=0.05,{ }^{*} P=0.01,{ }^{*} * P=0.001\right)$.

\section{Results}

\section{Normal pregnant as well as pregnant mice suffering from pregnancy disturbances display strong suppression of B cell lymphopoiesis}

Suppression of B lymphopoiesis in the BM during pregnancy has been previously demonstrated and was postulated to represent a physiological mechanism

Table 2 B cell subsets analyzed with their corresponding markers.

\begin{tabular}{|c|c|c|}
\hline Phenotype & B cell subset & Organ \\
\hline B220 ${ }^{\text {int }} \operatorname{slg}^{-}$ & Pre/pro & Bone marrow \\
\hline B220 ${ }^{\text {int }}$ slgM $^{-} \mathrm{CD}^{-} 3^{-}$ & Pre & Bone marrow \\
\hline $\mathrm{B} 220^{\mathrm{int}} \mathrm{slgM}^{-} \mathrm{CD} 43^{+}$ & Pro & Bone marrow \\
\hline B220 ${ }^{\text {int }}$ slgM $^{+}$ & Immature & Bone marrow \\
\hline $\mathrm{B}^{2} 20^{+}$slgM $^{+}$ & Mature & Bone marrow \\
\hline $\mathrm{B} 220^{+} \mathrm{CD} 23^{\mathrm{lo}} \mathrm{CD} 21^{\mathrm{hi}}$ & $M Z$ & Spleen \\
\hline $\mathrm{B} 220^{+} \mathrm{CD} 23^{\mathrm{hi}} \mathrm{CD} 21 / 35^{\mathrm{int}}$ & $\mathrm{FO}$ & Spleen \\
\hline $\mathrm{B} 220^{+} \mathrm{CD} 21^{+}$ & Mature & Para-aortic lymph node \\
\hline
\end{tabular}

tending to reduce the occurrence of autoreactive B cells, thus preventing pregnancy failures (Medina et al. 1993, Muzzio et al. 2014). Here, using an animal model of immune-mediated pregnancy disturbances, we observed that both pregnant mice undergoing normal pregnancies and those naturally suffering from pregnancy disturbances display significantly lower numbers of $\mathrm{B}_{22} 20^{\mathrm{lo}} \mathrm{slgM}^{-}$pre- and pro-B cells as well as $\mathrm{B}^{2} 20^{+} \mathrm{slgM}^{-}$immature B cells in the BM as compared to non-pregnant control mice (Fig. 1A). Further analysis showed that a number of $\mathrm{B} 220^{\mathrm{lo}} \mathrm{IgM}{ }^{-} \mathrm{CD}^{-} 3^{-}$pre-B cells as well as $\mathrm{B} 220^{\mathrm{lo}} \mathrm{IgM}^{-} \mathrm{CD}^{-} 3^{+}$pro-B cells were significantly decreased in the $\mathrm{BM}$ of normal pregnant mice and pregnant mice naturally suffering from pregnancy disturbances compared to non-pregnant animals

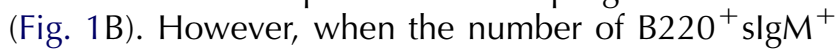
mature $B$ cells was evaluated, a significant reduction was observed in the BM of pregnant mice suffering from pregnancy disturbances compared to non-pregnant control mice (Fig. 1A). Pregnant mice undergoing normal pregnancies showed a slight, but not significant, reduction in the number of $\mathrm{B}_{2} 20^{+}$slgM $^{+}$mature $\mathrm{B}$ cells compared to non-pregnant mice (Fig. 1A). In summary, $\mathrm{B}$ cell lymphopoiesis is reduced in the BM of pregnant mice regardless of pregnancy outcome.

\section{$M Z B$ cells are expanded in the spleen of normal pregnant mice but not in those suffering from pregnancy disturbances}

In a previous study, we demonstrated that MZ B cell numbers were expanded in the spleen during pregnancy and proposed that this could be a compensatory mechanism launched to balance the lower influx of B cells observed in this tissue, thus maximizing the capacity of the maternal immune system to control pathogens (Muzzio et al. 2014). To test this hypothesis, we began analyzing the number of total $\mathrm{B}$ cells in the spleen of normal pregnant as well as pregnant mice naturally suffering from pregnancy disturbances and observed that, regardless of pregnancy outcome, pregnant mice showed a significantly lower number of total B220 ${ }^{+}$B cells in the spleen compared to non-pregnant control animals (Fig. 2A). These results prompted us to further investigate whether the distribution of main populations of mature B cells, namely, follicular and MZ B cells, in the spleen could also be altered during normal and pathological pregnancies. Indeed, we observed that the number of $\mathrm{B} 220^{+} \mathrm{CD} 23^{\text {hi }} \mathrm{CD} 21^{\text {int }}$ FO B cells was significantly decreased $(12.63 \pm 0.44 \times$ $10^{6}$ cells to $10.56 \pm 0.78 \times 10^{6}$ cells) in normal pregnant mice compared to non-pregnant controls (Fig. 2B). Diminution of FO B cell numbers was even more pronounced in pregnant mice suffering from pregnancy disturbances $\left(12.63 \pm 0.44 \times 10^{6}\right.$ cells to $9.49 \pm 0.61 \times$ $10^{6}$ cells; Fig. $2 \mathrm{~B}$ ). Interestingly, unlike FO B cells, the number of $\mathrm{B} 220^{+} \mathrm{CD} 23^{\mathrm{lo}} \mathrm{CD} 21^{\mathrm{hi}} \mathrm{MZ} B$ cells was 
A
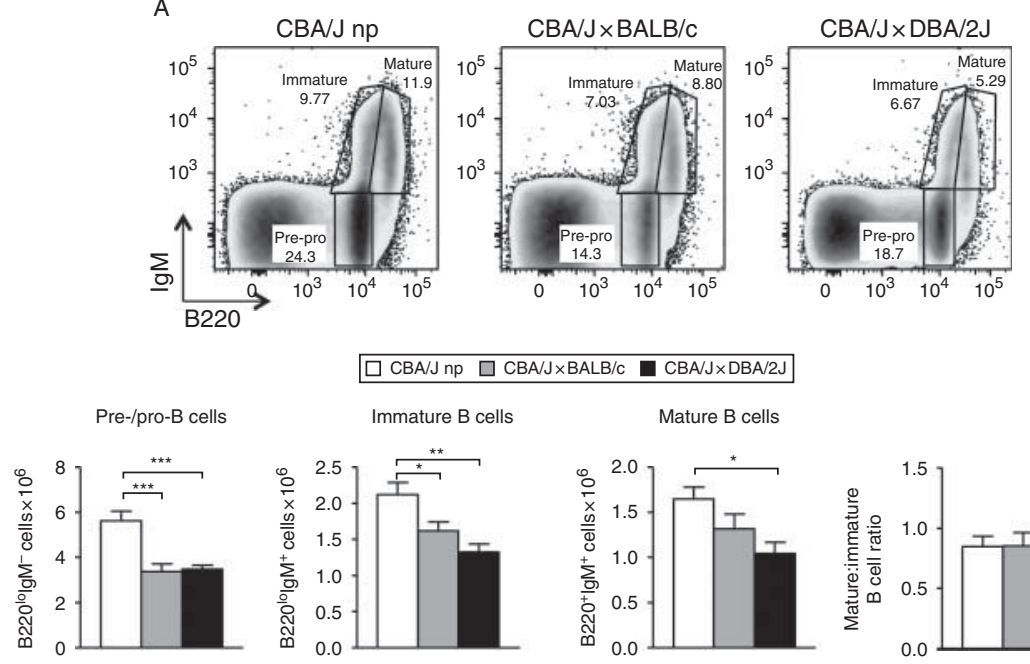

\section{$\square \mathrm{CBA} / \mathrm{Jnp} \quad \square \mathrm{CBA} / \mathrm{x}$} Mature B cells

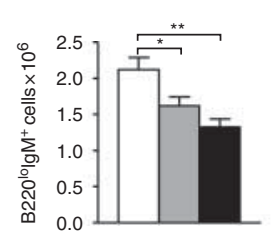

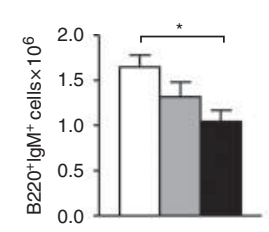

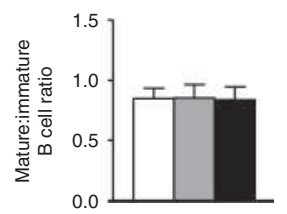

B

Figure $1 \mathrm{~B}$ cell lymphopoiesis is strongly reduced in normal pregnant as well as in pregnant mice suffering pregnancy failures. (A) Representative density plots displaying percentage and gating strategy used to analyze the population of pre- and pro- $\left(\mathrm{B}_{22} 2 \mathrm{lo}^{\mathrm{o}} \mathrm{slgM}^{-}\right)$, immature $\left(\right.$ B220 ${ }^{\mathrm{lo}}$ slgM $^{+}$) and mature $\left(\right.$B220 ${ }^{+}$slgM $^{+}$) B cells in the bone marrow (BM) of non-pregnant (np) CBA/J mice, BALB/c-mated CBA/J females (normal pregnant combination) and DBA/2Jmated CBA/J females (pregnant mice suffering pregnancy disturbances). Bar graphs show quantification of total numbers of pre- and pro-, immature and mature $B$ cells as well as the ratio of mature vs immature B cells in the BM of nonpregnant CBA/J $(n=13), \mathrm{BALB} / \mathrm{c}-$ mated normal pregnant mice $(n=12)$ and DBA/2J-mated CBA/J females (mice suffering pregnancy disturbances) $(n=10)$. (B) Density plots showing percentage and gating strategy applied to differentiate pro- $\mathrm{B}$ cells $\left(\mathrm{B}_{22} 2 \mathrm{Io}^{\mathrm{Ig}} \mathrm{gM}^{-} \mathrm{CD}^{+} 3^{+}\right)$from pre-B cells $\left(\mathrm{B} 220^{\mathrm{lo}} \mathrm{IgM}^{-} \mathrm{CD}^{-} 3^{-}\right.$) in the BM of non-pregnant $\mathrm{CBA} / \mathrm{J}$ mice, BALB/C-mated CBA/J and DBA/2Jmated CBA/J females. Bar graphs show quantification of total numbers of pre- and pro-B cells in the BM of non-pregnant CBA/J $(n=13), \mathrm{BALB} /$ c-mated $(n=12)$ and DBA/2J-mated CBA/J $(n=10)$ females. Data are expressed as mean \pm S.E.M. ${ }^{*} P<0.05,{ }^{* *} P<0.01,{ }^{* * *} P<0.001$ as analyzed by the one-way ANOVA, followed by a Tukey multiple comparison test.

significantly higher in normal pregnant mice compared to non-pregnant animals and pregnant mice suffering from pregnancy disturbances $(3.02 \pm 0.312-2.14 \pm 0.16$ and $1.31 \pm 0.18 \times 10^{6}$ cells respectively; Fig. $2 \mathrm{~B}$ ). We have additionally performed the ratio between $M Z$ and FO B cells and observed a clear preponderance of MZ over FO B cells in the spleen of normal pregnant mice compared to non-pregnant and pregnant mice suffering from pregnancy disturbances (Fig. 2B). In summary, normal pregnant mice, but not pregnant females, suffering from pregnancy disturbances exhibited an expansion of the $M Z B$ cells compartment despite $B$ cell lymphopenia observed in the spleen.

\section{Enhanced levels of MZ-produced immunoglobulin in serum of normal pregnant mice}

Having observed that a number of $M Z B$ cells were increased in the spleen of normal pregnant mice even though total B cell influx was reduced in this tissue, we next focused on the functionality of these cells in terms of antibody production. As shown in Fig. 3, serum levels of $\lg M$, the most prominent $M Z B$ cell-produced immunoglobulin (Guinamard et al. 2000, Martin \& Kearney 2002), were significantly higher in the serum of normal pregnant mice compared to non-pregnant control animals as well as pregnant mice suffering from pregnancy disturbances. Similarly, serum levels of IgA, another immunoglobulin classically produced by MZ B cells (Martin \& Kearney 2002, Cerutti et al. 2013), were also significantly higher in the serum of normal pregnant mice compared to non-pregnant control mice. When compared to pregnant mice having pathological pregnancies, the levels of $\lg A$ in the serum of normal pregnant mice were slightly, but not significantly, higher (Fig. 3). We have additionally analyzed the levels of $\operatorname{lgG} 1, \lg \mathrm{g} 2 \mathrm{a}, \operatorname{lgG} 3$ and $\lg \mathrm{E}$ and observed that all of these immunoglobulin were barely, not significantly, augmented in the serum of normal pregnant mice as compared to non-pregnant and pregnant mice suffering from 

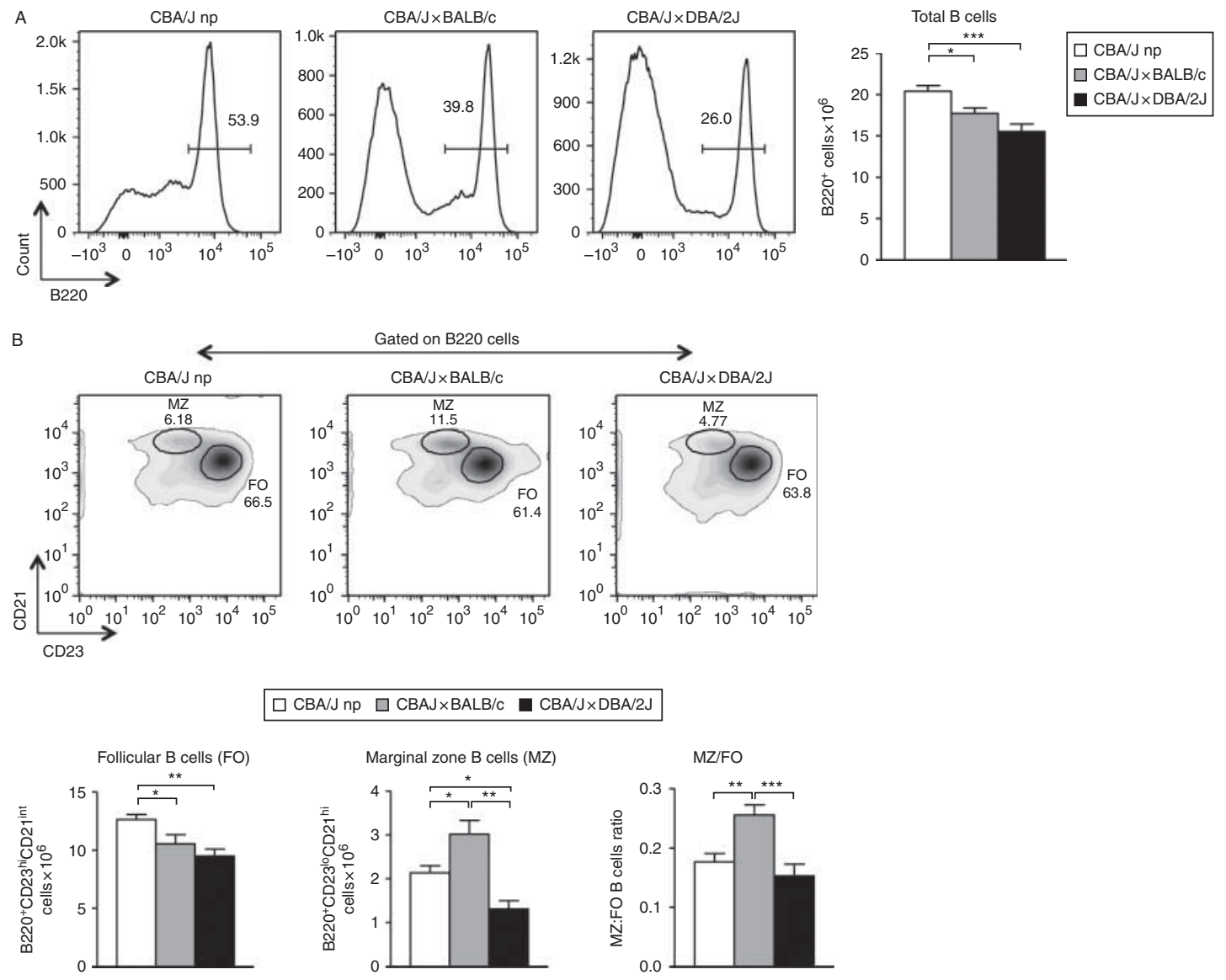

Figure $2 \mathrm{Marginal}$ zone B cell numbers are increased in the spleen of normal pregnant mice. (A) Representative histograms showing the expression of B220 in splenocytes from non-pregnant (np) CBA/J as well as BALB/c-mated CBA/J females (normal pregnant combination) and DBA/2J-mated CBA/J females (pregnant mice suffering pregnancy disturbances). Numbers on histogram indicate the percentage of B220 ${ }^{+}$cells. Bar graph shows total numbers of B220 B cells in the spleen of non-pregnant CBA/J $(n=13)$, BALB/c-mated CBA/J $(n=12)$ and DBA/2J-mated CBA/J $(n=10)$ females. (B) Representative density plots displaying percentage and gating strategy used to analyze $\mathrm{B} 220^{+} \mathrm{CD} 23^{\text {hi }} \mathrm{CD} 21^{\text {int }}$ follicular (FO) B cells (low right circle) and $\mathrm{B} 220^{+} \mathrm{CD} 23^{\mathrm{lo}} \mathrm{CD} 21^{\text {hi }}$ marginal zone (MZ) B cells (upper left circle). Bar graphs show total number of FO and MZ B cells as well as the ratio between $\mathrm{MZ}$ and FO B cells in the spleen of non-pregnant CBA/J $(n=13), \mathrm{BALB} / \mathrm{c}-$ mated CBA/J $(n=12)$ and DBA/2J-mated CBA/J $(n=10)$ females. Data are expressed as mean \pm S.E.M. ${ }^{*} P<0.05,{ }^{* *} P<0.01,{ }^{* * *} P<0.001$ as analyzed by the one-way ANOVA, followed by a Tukey multiple comparison test.

pregnancy disturbances. In summary, normal pregnancy seems to be associated with an enhanced production of immunoglobulin by MZ B cells.

\section{Reduced levels of BAFF in normal pregnant as well as pregnant mice suffering from abortions}

Taking into account the fact that we have previously described a reduction in the levels of BAFF during normal pregnancies in mice (Muzzio et al. 2014), we next wondered whether this is also true for pregnant animals suffering from immune-mediated pregnancy disturbances. As expected, we observed a significant reduction in the levels of BAFF in the serum of normal pregnant mice compared to non-pregnant control animals (from $8745 \pm 312.1$ to $7622 \pm 284.8 \mathrm{pg} / \mathrm{ml}$; Fig. 4). Interestingly, the reduction in the levels of BAFF was even more pronounced in the case of pregnant mice undergoing pregnancy disturbances compared to non-pregnant control animals (from $8745 \pm 312.1$ to $6959 \pm 283.4 \mathrm{pg} / \mathrm{ml}$; Fig. 4). There were no significant differences in the levels of BAFF between normal pregnant mice and those suffering from pregnancy failures (Fig. 4).

\section{Increased number of mature B cells in the lymph nodes draining the uterus of normal pregnant as well as pregnant mice suffering from abortions}

It has been previously demonstrated that the proportion (Newport \& Carter 1983) as well as the total number of B cells (Muzzio et al. 2014) in the para-aortic lymph nodes (PLN) draining the uterus is increased during pregnancy. In this study we first confirmed this by showing that 

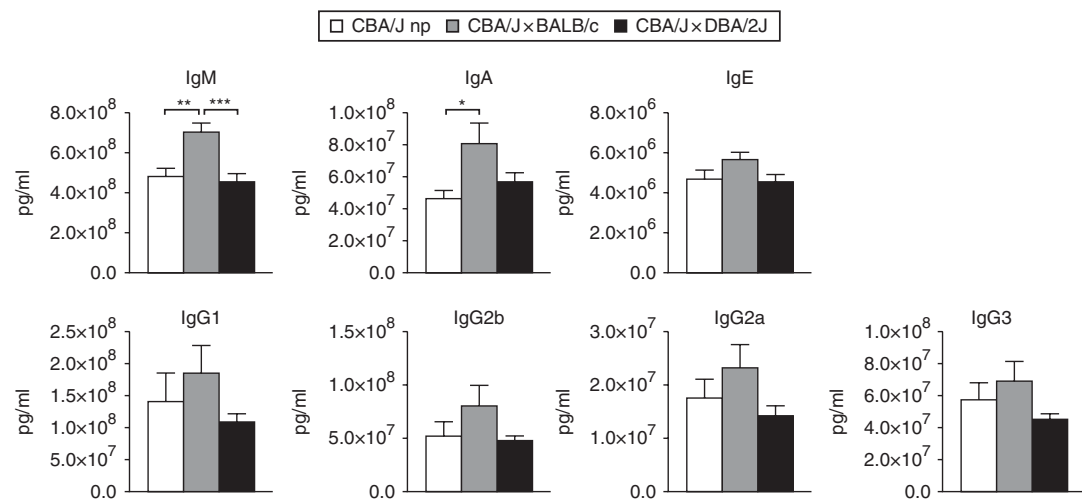

Figure 3 Levels of immunoglobulin in serum of non-pregnant and pregnant mice. Concentrations of $\lg \mathrm{M}, \lg \mathrm{A}, \lg \mathrm{G} 1, \lg \mathrm{G} 2 \mathrm{~b}, \lg \mathrm{g} 3, \lg \mathrm{G} 2 \mathrm{a}$ and $\lg \mathrm{E}$ were evaluated in the serum of non-pregnant $(n p)$ $\mathrm{CBA} / \mathrm{J}$ females $(n=13)$ as well as BALB/c-mated (normal pregnant mice) $(n=12)$ and DBA/2Jmated CBA/J females (suffering pregnancy disturbances) $(n=10)$. Data are expressed as mean \pm S.E.M. $* P<0.05,{ }^{* *} P<0.01,{ }^{* * *} P<0.001$ as analyzed by the one-way ANOVA, followed by a Tukey multiple comparison test. normal pregnant mice displayed a significantly higher number of $\mathrm{B} 220^{+} \mathrm{CD} 21^{+}$mature $\mathrm{B}$ cells in PLN compared to non-pregnant mice (Fig. 5) and then extended these results to show that pregnant animals suffering from abortions also displayed a significantly higher number of $\mathrm{B} 220^{+} \mathrm{CD} 21^{+}$mature B cells in PLN compared to non-pregnant mice (Fig. 5). In summary, during pregnancy, the number of mature B cells is increased in the uterine-draining lymph nodes regardless of pregnancy outcome.

\section{Discussion}

We demonstrated in this study that normal pregnant mice but not those suffering from immune-mediated pregnancy failure can compensate for the strong reduction of B cell lymphopoiesis as well as splenic B cell lymphopenia occurring during gravidity by inducing mechanisms tending to favor the presence of the preactivated MZ B cells and the production of natural protective antibodies. Successful pregnancy in mammals relies on the capacity of the maternal immune system to simultaneously tolerate the semi-allogeneic fetus and protect the mother against potential infections (Arck \& Hecher 2013). This requires strong and highly regulated adaptations of maternal immunity. Suppression of B cell lymphopoiesis has been postulated to be one of these mechanisms, representing an evolutionarily acquired strategy tending to reduce the occurrence of autoreactive $B$ cells that might recognize fetal structures and put pregnancy at risk (Medina et al. 1993, Ait-Azzouzene et al. 1998, Muzzio et al. 2014). However, the fact that a lower number of B cells are being produced during this critical period of time can also compromise the capacity of the maternal immune system to fight pathogens. Hence, it becomes evident that additional adaptations are demanded. Indeed, using a mouse model of immune-mediated pregnancy failure, we demonstrated in this study that the suppression of B cell lymphopoiesis seems not to be directly associated with pregnancy outcome as both normal pregnant and pregnant mice suffering from immune-mediated pregnancy disturbances showed a significant reduction of B cell lymphopoiesis in their BM. These results prompted us to further investigate whether, independently of a direct effect on pregnancy well-being, the reduction of B cell lymphopoiesis during gravidity might affect B cell differentiation in the periphery. To analyze this, we then focused on the spleen, which is the tissue in which immature $\mathrm{B}$ cells arriving from the BM become either $\mathrm{FO}$ or $M Z B$ cells, the most prominent mature $B$ cell in the body (Monroe \& Dorshkind 2007, Allman \& Pillai 2008). These two B cell subtypes have different but complementary functions (Monroe \& Dorshkind 2007, Allman \& Pillai 2008). In a process that lasts $\sim 5$ days after encountering an antigen, FO B cells produce $\mathrm{T}$ celldependent high affinity antibodies, mainly IgG subtypes (Cerutti et al. 2013). In contrast, due to their preactivated phenotype, shortly after encountering an antigen, MZ B cells produce, in a T cell independent fashion, low affinity antibodies that are crucial for controlling the first wave of infection (Martin et al. 2001, Colino et al. 2002). In the context of pregnancy,

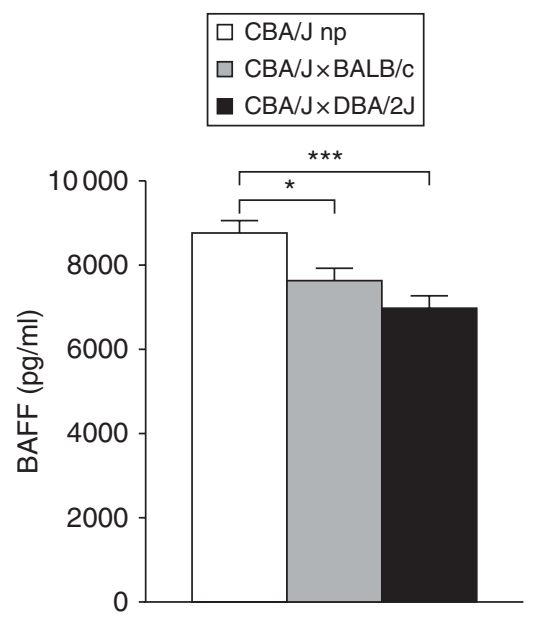

Figure 4 Levels of B cell-activating factor (BAFF) in the serum of nonpregnant and pregnant mice. The concentration of BAFF was evaluated in serum of non-pregnant CBA/J females $(n=13)$ as well as BALB/ c-mated CBA/J normal pregnant mice $(n=12)$ and DBA/2J-mated CBA/J pregnant mice suffering from pregnancy disturbances $(n=10)$. Data are expressed as mean \pm s.E.M. $* P<0.05,{ }^{* * *} P<0.001$ as analyzed by the one-way ANOVA, followed by a Tukey multiple comparison test. 

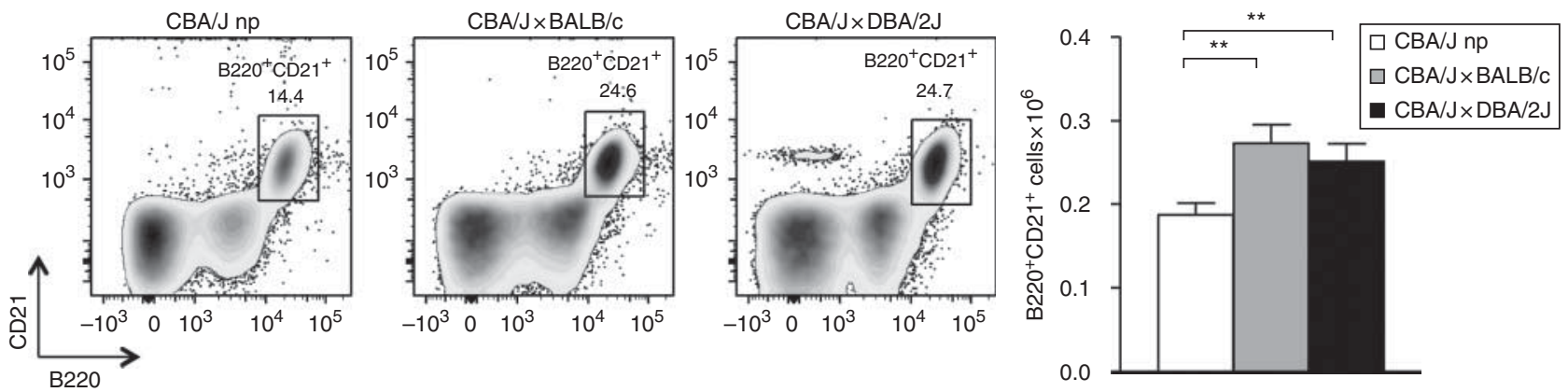

Figure 5 Mature B cell numbers are expanded in the lymph nodes draining the uterus during pregnancy. Representative density plots displaying the percentage as well as gating strategy used to analyze $\mathrm{B} 220^{+} \mathrm{CD} 21^{+}$mature B cells in the para-aortic lymph nodes of non-pregnant (np) $\mathrm{CBA} / \mathrm{J}$ females $(n=13)$ as well as BALB/C-mated CBA/J normal pregnant mice $(n=12)$ and DBA/2J-mated CBA/J pregnant mice suffering from pregnancy disturbances $(n=10)$. Data are expressed as mean \pm s.E.M. ${ }^{* *} P<0.01$ as analyzed by the one-way ANOVA, followed by a Tukey multiple comparison test.

we have recently demonstrated that, although the total number of B cells as well as FO B cells is reduced, the number of pre-activated $M Z B$ cells is increased in the spleen of pregnant mice (Muzzio et al. 2014). Based on these results we proposed that the expansion of the preactivated MZ B cell compartment may represent an acquired mechanism launched to compensate the lower influx of B cells into the spleen, thus maximizing the capacity of the maternal immune system to control infections (Muzzio et al. 2014). Indeed, we confirm here that normal pregnant mice, but not those suffering from immune-mediated pregnancy failures, were able to induce an expansion of $M Z B$ cells even in the presence of a splenic B cell lymphopenia. Interestingly, it has been previously proposed that the high incidence of pregnancy failure naturally occurring in the DBA/2J-mated $\mathrm{CBA} / \mathrm{J}$ females is correlated with their higher susceptibility to environmental antigens (Hamilton \& Hamilton 1987) and can be prevented or reduced by transferring natural antibodies from normal pregnant BALB/c-mated CBA/J females (Chaouat et al. 1985, Hamilton \& Hamilton 1987). Natural antibodies are crucial components of the first-line defense against invading pathogens and also have a homeostatic role in regulating the clearance of necrotic and apoptotic cells, thus preventing inflammatory reactions (SchwartzAlbiez et al. 2009). They are produced by MZ and B-1a B cells in the absence of antigen stimulation (Kaveri et al. 2012, Silverman et al. 2013). The majority of natural antibodies consist of IgM with a smaller proportion of IgA subtype (Schwartz-Albiez et al. 2009). Notably, in addition to an expansion of MZ B cells, we observed significantly higher levels of IgM and, in a minor extent, IgA, in the serum of normal pregnant mice compared to non-pregnant or mice suffering pregnancy disturbances. Hence, it becomes evident that pregnancy well-being relies on a proper expansion and activation of the MZ B cell compartment.

We next concentrated on investigating factors involved in the differentiation of immature B cells into
MZ B cells or FO B cells. The TNF family ligand BAFF/Blys is an essential growth factor for B cell development and maintenance (Schiemann et al. 2001, Schneider et al. 2001, Pillai \& Cariappa 2009). We have previously shown that pregnant mice display lower levels of BAFF as compared to non-pregnant animals (Muzzio et al. 2014). Here we first confirmed and then extended these results by demonstrating that mice suffering from immune-mediated pregnancy failures showed a more pronounced reduction in the levels of BAFF when compared to non-pregnant animals. However, we did not observe significant differences in the levels of BAFF between normal pregnant mice and those suffering pregnancy disturbances. Hence, it becomes evident that other factors rather than BAFF control the dynamic of MZ B cells during gravidity. Pregnancy well-being has been classically associated with a shift toward a Th2 profile, which favors the development of humoral over cellular immunity (Saito et al. 2010). In this regard, our results concerning elevated levels of $\operatorname{Ig} M$ and $\operatorname{Ig} \mathrm{A}$ - immunoglobulin more likely produced by $M Z B$ cells - in serum of normal pregnant mice seem to at least partially support this idea. Noteworthy, levels of IgG, a FO B cell-produced immunoglobulin, did not show significant differences among the groups. $T$ follicular helper (Tfh) cells are known to be crucial in regulating antibody production by controlling isotype class switching and plasma cell differentiation, a process involving FO B cells (Crotty 2011). Furthermore, Tfh cells are also able to induce MZ B cell apoptosis (Tortola et al. 2010). Taking this into account, our results might suggest a lack of Tfh activity during pregnancy, favoring a survival of MZ B cells and a shift toward $T$ cell independent antibody response.

In summary, we have demonstrated in this study that the suppression of B cell lymphopoiesis occurring during gravidity in mice does not seem to directly affect pregnancy outcome. However, it has a strong impact on the influx of B cells into the spleen, as a splenic B cell lymphopenia was observed in pregnant mice. Notably, 
normal pregnant animals, but not those suffering from immune-related pregnancy failures, could compensate $B$ cell lymphopenia by inducing an expansion of the preactivated MZ B cells and the production of natural antibodies, thus maximizing the capacity of immune defense and avoiding undesired inflammatory reactions. Whether the increased number of MZ B cells, observed during normal pregnancy, is due to a preferential differentiation process of this B cell lineage or simply an expansion of pre-existing MZ B cells in the spleen due to an increased proliferation or decreased apoptosis rate should be addressed in the future.

Our results clearly highlight the fundamental role of the $M Z B$ cells in the intricate balance between immune suppression and activation launched during pregnancy to simultaneously tolerate the presence of the semiallogeneic fetus and ensure a proper defense of the mother against pathogens. Further studies are required in order to identify factors controlling this phenomenon.

\section{Declaration of interest}

The authors declare that there is no conflict of interest that could be perceived as prejudicing the impartiality of the research reported.

\section{Funding}

This study was supported by a grant from the Fritz Thyssen Foundation to $\mathrm{F} J$ (Az. 10.12.2.155) and intramural funding from Greifswald University.

\section{Acknowledgements}

We thank Kai Masur and Liane Kantz from the Leibniz Institute for Plasma Science and Technology, Plasmalifescience (INP Greifswald), for their kind and valuable assistance in the performance of the multiplex assay.

\section{References}

Ahmed A, Singh J, Khan Y, Seshan SV \& Girardi G 2010 A new mouse model to explore therapies for preeclampsia. PLOS ONE 5 e13663. (doi:10.1371/journal.pone.0013663)

Ait-Azzouzene D, Gendron MC, Houdayer M, Langkopf A, Burki K, Nemazee D \& Kanellopoulos-Langevin C 1998 Maternal B lymphocytes specific for paternal histocompatibility antigens are partially deleted during pregnancy. Journal of Immunology 161 2677-2683.

Allman D \& Pillai S 2008 Peripheral B cell subsets. Current Opinion in Immunology 20 149-157. (doi:10.1016/j.coi.2008.03.014)

Arck PC \& Hecher K 2013 Fetomaternal immune cross-talk and its consequences for maternal and offspring's health. Nature Medicine 19 548-556. (doi:10.1038/nm.3160)

Cerutti A, Cols M \& Puga I 2013 Marginal zone B cells: virtues of innate-like antibody-producing lymphocytes. Nature Reviews. Immunology 13 118-132. (doi:10.1038/nri3383)

Chaouat G, Kolb JP, Kiger N, Stanislawski M \& Wegmann TG 1985 Immunologic consequences of vaccination against abortion in mice. Journal of Immunology 134 1594-1598.
Chu H, Awasthi A, White GC II, Chrzanowska-Wodnicka M \& Malarkannan S 2008 Rap1b regulates B cell development, homing, and T cell-dependent humoral immunity. Journal of Immunology $\mathbf{1 8 1}$ 3373-3383. (doi:10.4049/jimmunol.181.5.3373)

Clark DA, Chaput A \& Tutton D 1986 Active suppression of host-vs-graft reaction in pregnant mice. VII. Spontaneous abortion of allogeneic CBA/J $x$ DBA/2 fetuses in the uterus of CBA/J mice correlates with deficient nonT suppressor cell activity. Journal of Immunology 136 1668-1675.

Colino J, Shen Y \& Snapper CM 2002 Dendritic cells pulsed with intact Streptococcus pneumoniae elicit both protein- and polysaccharidespecific immunoglobulin isotype responses in vivo through distinct mechanisms. Journal of Experimental Medicine 195 1-13. (doi:10.1084/ jem.20011432)

Crotty S 2011 Follicular helper CD4 T cells (TFH). Annual Review of Immunology 29 621-663. (doi:10.1146/annurev-immunol-031210101400)

Faas MM, Spaans F \& De Vos P 2014 Monocytes and macrophages in pregnancy and pre-eclampsia. Frontiers in Immunology 5298. (doi:10.3389/fimmu.2014.00298)

Guinamard R, Okigaki M, Schlessinger J \& Ravetch JV 2000 Absence of marginal zone B cells in Pyk-2-deficient mice defines their role in the humoral response. Nature Immunology 1 31-36. (doi:10.1038/76882)

Hamilton MS \& Hamilton BL 1987 Environmental influences on immunologically associated spontaneous abortion in CBA/J mice. Journal of Reproductive Immunology 11 237-241. (doi:10.1016/01650378(87)90060-X)

Hardy RR 2003 B-cell commitment: deciding on the players. Current Opinion in Immunology 15 158-165. (doi:10.1016/S0952-7915(03) 00012-8)

Hardy RR \& Hayakawa K 1991 A developmental switch in B lymphopoiesis. PNAS 88 11550-11554. (doi:10.1073/pnas.88.24.11550)

Kanasaki K \& Kalluri R 2009 The biology of preeclampsia. Kidney International 76 831-837. (doi:10.1038/ki.2009.284)

Kaveri SV, Silverman GJ \& Bayry J 2012 Natural IgM in immune equilibrium and harnessing their therapeutic potential. Journal of Immunology $\mathbf{1 8 8}$ 939-945. (doi:10.4049/jimmunol.1102107)

Martin F \& Kearney JF 2002 Marginal-zone B cells. Nature Reviews. Immunology 2 323-335. (doi:10.1038/nri799)

Martin F, Oliver AM \& Kearney JF 2001 Marginal zone and B1 B cells unite in the early response against T-independent blood-borne particulate antigens. Immunity 14 617-629. (doi:10.1016/S1074-7613(01)00129-7)

Medina KL, Smithson G \& Kincade PW 1993 Suppression of B lymphopoiesis during normal pregnancy. Journal of Experimental Medicine 178 1507-1515. (doi:10.1084/jem.178.5.1507)

Monroe JG \& Dorshkind K 2007 Fate decisions regulating bone marrow and peripheral B lymphocyte development. Advances in Immunology 95 1-50. (doi:10.1016/S0065-2776(07)95001-4)

Muzzio DO, Soldati R, Ehrhardt J, Utpatel K, Evert M, Zenclussen AC, Zygmunt M \& Jensen F 2014 B cell development undergoes profound modifications and adaptations during pregnancy in mice. Biological Reproduction 91 115. (doi:10.1095/biolreprod.114.122366)

Newport A \& Carter J 1983 Changes in T and B lymphocyte populations in the lymph nodes draining the uterus in pregnant mice. Journal of Reproduction \& Fertility 67 433-440. (doi:10.1530/jrf.0.0670433)

Parham P 2004 NK cells and trophoblasts: partners in pregnancy. Journal of Experimental Medicine 200 951-955. (doi:10.1084/jem.20041783)

Pillai S \& Cariappa A 2009 The follicular versus marginal zone B lymphocyte cell fate decision. Nature Reviews. Immunology 9 767-777. (doi:10.1038/nri2656)

Ruocco MG, Chaouat G, Florez L, Bensussan A \& Klatzmann D 2014 Regulatory T-cells in pregnancy: historical perspective, state of the art, and burning questions. Frontiers in Immunology 5 389. (doi:10.3389/ fimmu.2014.00389)

Saito S, Nakashima A, Shima T \& Ito M 2010 Th1/Th2/Th17 and regulatory T-cell paradigm in pregnancy. American Journal of Reproductive Immunology 63 601-610. (doi:10.1111/j.1600-0897.2010.00852.x)

Schiemann B, Gommerman JL, Vora K, Cachero TG, Shulga-Morskaya S, Dobles M, Frew E \& Scott ML 2001 An essential role for BAFF in the normal development of B cells through a BCMA-independent pathway. Science 293 2111-2114. (doi:10.1126/science.1061964)

Schneider P, Takatsuka H, Wilson A, Mackay F, Tardivel A, Lens S, Cachero TG, Finke D, Beermann F \& Tschopp J 2001 Maturation of 
marginal zone and follicular B cells requires B cell activating factor of the tumor necrosis factor family and is independent of B cell maturation antigen. Journal of Experimental Medicine 194 1691-1697. (doi:10.1084/jem.194.11.1691)

Schwartz-Albiez R, Monteiro RC, Rodriguez M, Binder CJ \& Shoenfeld Y 2009 Natural antibodies, intravenous immunoglobulin and their role in autoimmunity, cancer and inflammation. Clinical Experimental Immunology 158 (Suppl 1) 43-50. (doi:10.1111/j.1365-2249.2009.04026.x)

Segerer SE, Staib C, Kaemmerer U, Frambach T, Honig A, Dietl J \& Rieger L 2012 Dendritic cells: elegant arbiters in human reproduction. Current Pharmaceutical Biotechnology 13 1378-1384. (doi:10.2174/138920 112800784916)
Silverman GJ, Vas J \& Gronwall C 2013 Protective autoantibodies in the rheumatic diseases: lessons for therapy. Nature Reviews. Rheumatology 9 291-300. (doi:10.1038/nrrheum.2013.30)

Tortola L, Yadava K, Bachmann MF, Müller C, Kisielow J \& Kopf M 2010 IL21 induces death of marginal zone B cells during chronic inflammation. Blood 116 5200-5207. (doi:10.1182/blood-2010-05-284547)

Received 19 June 2015

First decision 29 July 2015

Revised manuscript received 13 October 2015

Accepted 21 October 2015 